\title{
Spline Surface Intersections Optimized for GPUs
}

\author{
Sverre Briseid ${ }^{1}$, Tor Dokken ${ }^{1,2}$, Trond Runar Hagen ${ }^{1,2}$, \\ and Jens Olav Nygaard ${ }^{1}$
}

1 SINTEF, Dept. of Applied Math., P.O. Box 124 Blindern, N-0314 Oslo, Norway

2 Centre of Mathematics for Applications (CMA), University of Oslo, Norway

$\{$ sbr, tdo, trr, jnygaard\}@sintef.no

http://www. sintef.no/gpgpu

\begin{abstract}
A commodity-type graphics card with its graphics processing unit (GPU) is used to detect, compute and visualize the intersection of two spline surfaces, or the self-intersection of a single spline surface. The parallelism of the GPU facilitates fast and efficient subdivision and bounding box testing of smaller spline patches and their corresponding normal subpatches. This subdivision and testing is iterated until a prescribed level of accuracy is reached, after which results are returned to the main computer. We observe speedups up to 17 times relative to a contemporary 64 bit CPU.
\end{abstract}

\section{Introduction}

We can divide most surface intersections into three categories: 1) self-intersections for which the normal degenerates to zero length, 2) self-intersections for which this does not happen, and 3) intersections between two surfaces, resulting in an intersection curve, see Figure 1. There are a number of other kinds of intersections, more or less degenerate, like intersections in points, intersections with coincident parts of the surface(s), etc. See [5] for more information about intersections.

Detecting and finding intersections is particularly important in all computer aided design (CAD) systems, as well as in numerous other applications. In CAD systems, geometries are traditionally represented with the help of spline surfaces and spline curves. For an introduction to splines, see e.g., [2]. With the advent of fast and programmable GPUs offering 32 bit floating point precision, a natural course of action is to use the GPU for such geometric computations.

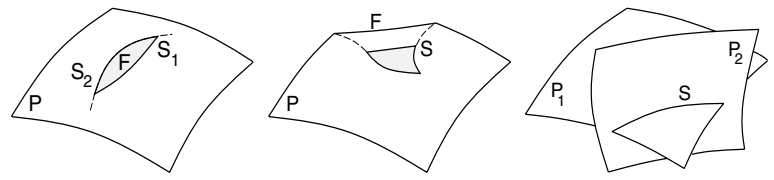

Fig. 1. From the left, a self-intersection with degenerate normals (a), a self-intersection with no degenerate normals (b) and an intersection of two surfaces resulting in a well defined intersection curve (c) 
The main limitations of the GPU are the single precision arithmetics, the limited inter-process communication, and the bottleneck in the passing of results back to the main computer from the graphics card. By adapting our algorithms to the GPU, we have maneuvered around these obstacles. The processing of geometry on the GPU is not farfetched, since the GPU is designed not only to render geometry, but also to some degree process it, even though this is focused around the processing of triangles.

\section{Background}

Current CAD-technology is built on the STEP-standard (ISO 10303) from the early 1990s, and is consequently based on the computer performance at that time. Volume objects in CAD are described by the outer and possibly inner shells limiting the volume. A shell is described by a surface patchwork. Degree 1 and 2 algebraic surfaces, i.e., planes, spheres, cones and cylinders are central in CADsystems. More complex sculptured shapes are represented by piecewise rational parametric surfaces using non-uniform rational B-spline surfaces (NURBS).

Closed forms can be found for the intersection of surfaces of algebraic degrees 1 and 2. However, for intersections of surfaces of higher algebraic degree, numerical methods have to be used. CAD surface intersection until recently only worked well for transversal intersections, where the normals of the surfaces are non-parallel along the intersection curve [9]. If the normal fields of two surfaces do not intersect then Sinha's theorem [7] states that the surfaces do not have a closed intersection loop. Thus recursive subdivision can be used to create subsurfaces in regions with potential closed intersection loops and detect these (loop destruction) [8]. For singular intersections where the surface normals are parallel along the intersection curve the theorem does not apply. For near singular intersections where the surface normals are near parallel along the intersection curve, the theorem often does not help much, as normal fields are in general approximated before overlap is tested [3, 6].

To better solve the singular and near singular surface intersection problems, as well as attempt to solve the surface self-intersection challenge, the idea of approximate implicitization was introduced [1. In the EU IST FET-Open GAIA projects (2000-2005) surface intersection and self-intersection algorithms were developed following these ideas. However, performance of these new combined recursive and approximate implicit intersection algorithms is not as good as required for industrial use. Thus the idea was born to use the GPU for "naive" extensive subdivision to establish guaranteed intersection conjectures that can guide the recursion strategy used in the CPU-based algorithms.

\section{Numerical Methods and Implementation on the GPU}

Given a spline surface, it can contain any number and combination of the intersections in Figure 1. If we find an intersection in a subpart of the surface, this does not exclude other intersections in the same subpart. We will now discuss 


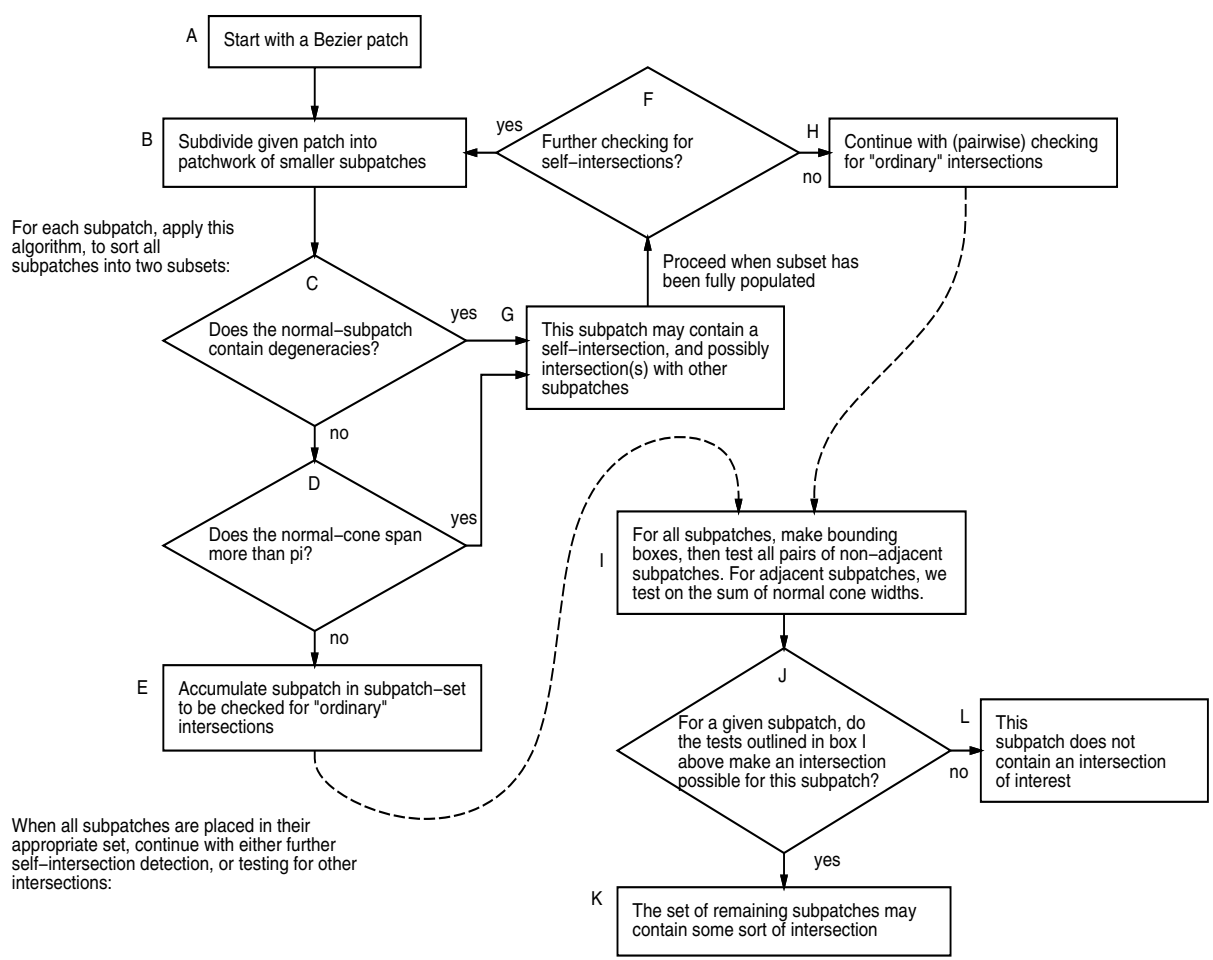

Fig. 2. The flowchart describing the processing of a Bezier-patch. Note that in stages $\mathrm{C}$ and $\mathrm{D}$, subpatches can be processed in parallel, and that subpatches are collected in sets in E, G and $\mathrm{H}$. In J, pairs of subpatches are processed in parallel, while sets of subpatches again are accumulated in $\mathrm{K}$ and L. Finally, any resulting subpatch in these sets may be further subdivided in $\mathrm{A}$, and the procedure repeated.

how to find the various kinds of intersections, with reference to the schematic flowchart in Figure 2.

\subsection{Splines and Spline Surfaces}

One special case of a spline surface is the Bezier patch. A Bezier patch of degree $d$, or order $k=d+1$, is a linear combination of $k^{2}$ basis functions, and can therefore be represented by a $k \times k$ matrix of coefficients. A larger spline surface can be subdivided into a set of Bezier patches, each of which can be further subdivided. The coefficient matrix for a patchwork of Bezier patches is a matrix of independent $k \times k$ sub-matrices of coefficients, which makes Bezier patches convenient building blocks in our spline-based GPU-algorithms.

Since we are interested in 3D surfaces, our spline surfaces will have 3D coefficients, or if we use rational spline surfaces (NURBS) 4D coefficients. In this paper, we focus on non-rational splines, but we note that in both cases the coefficients fit nicely into the RGBA-quadruples of GPU fragments. 


\subsection{Knot Insertion and Subdivision}

We have a spline surface $S(u, v)=\sum_{i, j=1}^{p} c_{i, j} N_{i}(u) N_{j}(v)=\left(\boldsymbol{N}^{T} C \boldsymbol{N}\right)(u, v)$, of degree $d$, where $\boldsymbol{N}^{T}=\left(N_{1}(\cdot), \ldots, N_{p}(\cdot)\right)$ consists of $p$ B-spline functions and $C$ is a $p \times p$ matrix of $3 \mathrm{D}$ coefficients. By giving all knots multiplicity $k$ by $k n o t$ insertion, we get a set of Bezier subpatches describing the same surface. For simplicity, and without loss of generalization, we use the same knot vectors for the two directions, resulting in the new representation

$$
S(u, v)=\left(\boldsymbol{N}^{T} C \boldsymbol{N}\right)(u, v)=\left(\overline{\boldsymbol{N}}^{T} D \overline{\boldsymbol{N}}\right)(u, v),
$$

where the new coefficients $D$ are given by $D=A C A^{T}$, and we have the relation $\boldsymbol{N}=A \overline{\boldsymbol{N}}$ between the new and old basis functions $\overline{\boldsymbol{N}}$ and $\boldsymbol{N}$. Here, $A$ is the knot insertion matrix corresponding to the insertion of the new knots. For more on splines and knot insertion, see again [2].

For $S$ itself a Bezier patch, we would typically subdivide it into $2^{2 n}$ new Bezier subpatches, by splitting the original knot vector interval into $2^{n}$ subintervals. We compute the accompanying knot insertion matrix $A$ on the $\mathrm{CPU}$ and pass it on as a texture together with $C$ to the GPU. The computation of the new coefficient matrix $D$ can then be done efficiently on the GPU using two passes for the two matrix multiplications in $D=(A C) A^{T}$. Notice that only the second of these multiplications, $A C$ multiplied with $A^{T}$, really does gain from the efficiency of the GPU, since the first multiplication only results in a matrix with order $\mathcal{O}\left(2^{n}\right)$ coefficients, while the latter produces $\mathcal{O}\left(2^{2 n}\right)$ coefficients. These shaders are quite simple, and have good "arithmetic to texture fetch" ratios for our cubic 3D Bezier patches.

We then test each of the subpatches for self-intersections and/or pairs of them for intersections and discard subpatches without any possible (self-)intersections.

This whole procedure can then be iterated. The result is a set of small patches which may contain intersections. These can be used as a starting point for more traditional intersection algorithms which are better suited for the CPU, or used directly for visualization. A subset of the three kinds of intersections may be computed by breaking off proper parts of the algorithm in Figure 2.

\subsection{Bounding Box Tests}

We look for intersections (not self-intersections) by subdividing patches and discarding subpatches that do not have rectangular bounding boxes that overlap any other than those of their neighbours. Note that the convex hull property for splines implies that the subpatches are contained in these boxes. This does not guarantee an intersection in the remaining subpatches, but we will never discard subpatches wrongly.

To avoid making an assumption about adjacent subpatches (in the parameter domain) not intersecting each other even though their bounding boxes always will, we do not use the bounding box test on such pairs, but instead compare the sum of their normal cone widths to $\pi$. The normal cone is a bounding box 

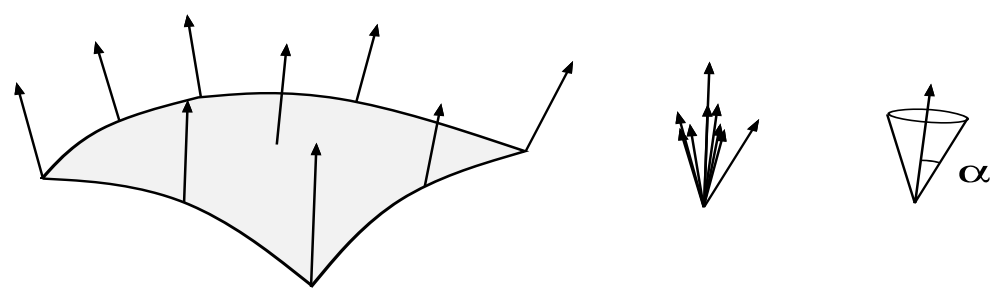

Fig. 3. From the left, a) a Bezier patch with normals indicated, b) the normals collected, and c) the normal cone with the angle/width $\alpha$ indicated

for the normals of a subpatch, specified as a direction and an angle, as shown in Figure 3. If the sum is smaller than $\pi$, the adjacent subpatches cannot intersect. (See Figure 2, boxes I and J.)

If we subdivide a Bezier patch into $2^{n} \times 2^{n}$ subpatches, we get $2^{4 n}$ ordered pairs of subpatches, including both subpatches paired to themselves and pairs of neighbouring subpatches. Given the subpatches organized in a $2^{n} k \times 2^{n} k$ coefficient matrix, as produced by the knot insertion of Section 3.2, we can easily form two matrices of size $2^{n} \times 2^{n}$ containing two opposite corners of the bounding boxes of the subpatches, on the GPU.

To compute the bounding boxes, we use the depth buffer to implement a fast maximum and minimum operator to be applied to all coefficients of all the subpatches. The bounding boxes are represented as two $2^{n} \times 2^{n}$ textures, that are afterward treated as $1 \mathrm{D}$ textures of length $2^{2 n}$. These are then used to form the tensor product of all pairs, and a new texture of size $2^{2 n} \times 2^{2 n}$ is used to store the boolean results of all the bounding box tests. Since this matrix is symmetric, we can eliminate half of the tests by rendering a triangle rather than a quad when running the corresponding shader on the GPU.

\subsection{Self-intersection Tests}

The subpatches making out the full Bezier patch can be independently checked for self-intersections. We do this by checking for degenerate, or "close to degenerate" normals, and checking the width of the normal cone.

Degenerate Normals. If we take a smooth surface without any intersections, and pull a part far away from any edges out and fold it back into itself, we get a self-intersecting surface as shown in Figure 1a. Here, a fold $F$ with the darkened underside of patch $P$ is shown. In the two intersection points $S_{1}$ and $S_{2}$ we have degenerate normals. Note that on the sides of the protruding fold $F$ we also have intersections like those in Figure 1b and 1 $\mathrm{c}$, in which the normals are not degenerate.

To test for degenerate normals, we form the normal surface of the patch, and subdivide this like the patch itself. The only difference is that if the surface patch has degree $d$, the normal surface will have degree $2 d-1$. From each subpatch of the normal surface, we find a bounding box for the coefficients, thus getting a bounding box for the normals. If this bounding box does not contain the origin 
(given some tolerance) the corresponding surface subpatch cannot contain any degenerate normal, and we can dismiss the subpatch as not having this particular kind of self-intersection. This corresponds to the test $C$ in Figure 2

We compute the normal surface of the $d$-degree Bezier patch on the CPU, and subdivide it on the GPU as described above in Section 3.2. Since the degree is $2 d-1$ we cannot use exactly the same shaders as for the Bezier patch itself, as we do not want the degree to be a variable. Rather, we use specialized shaders for each degree, but the algorithm is the same.

The test of degeneracy is then applied to this hull of all the subpatches, i.e., all the coefficients of the normal subpatches are compared to a specified small tolerance. This will result in a large matrix of boolean values, which can then be treated according to how we use it for specific applications. If further subdivison is to be performed, we can remove the irrelevant subpatches, and start the iteration again on the remaining subpatches. Or these can be returned to the CPU. A third use is to simply detect whether any of the normals at all are degenerate, this can be done very quickly with occlusion culling techniques.

Normal Cone Tests. Note that if the subpatch is to contain any self-intersections, we must have a normal cone with width greater than $\pi$. This test corresponds to the one depicted as test $D$ in the algorithm in Figure 2.

For these tests we make approximate normal cones that are not as "tight" as they could be, but faster to construct on the GPU. We average the coefficients of the normal subpatches, i.e., the subdivided normal patches, for the direction of the cones. The width of each cone is computed as the maximal deviation from this average to all normals on the subpatch. This is accomplished by iterating a very simple shader, in effect averaging, or blending, triples (the directions) and storing the largest angle.

\subsection{Tying It Together}

In Sections 3.1 to 3.4, we have seen how we take a spline surface as input, subdivide it into a larger set of Bezier subpatches, and perform tests on subpatches and pairs of such to determine whether certain intersections can be present. Depending on what tests we want to perform, we may adapt the algorithm in Figure 2 to our purpose. If we are interested in, e.g., only self-intersections of the kind producing degenerate normals, we iterate the sub-loop $A B C G F$. If we are interested in not only detecting the presence of self-intersections for which the normals do not disappear, but also finding them, we must follow a path in the flowchart ending in either $E$ or $H$, followed by the subpatch-pair testing $J$, resulting in a set of patches in $K$ that may contain intersections. Further processing of this set can be done either on the CPU, or the subpatches can be subdivided by iterating the algorithm from the top again. In the latter case, the currently slow returning of larger amounts of data from the GPU to the CPU can be avoided, the cost being that one must instead remove the uninteresting subpatches from the pool of such subpatches on the GPU. The authors are currently working on such an addition, following the ideas in [4]. 


\section{Results and Conclusions}

We have made use of the GPU to do computations that it was not primarily designed for. This means that the algorithms chosen are somewhat different from what have been used so far on more general computers, for the same purposes.

To fit the GPU architecture, the algorithm has been made less adaptive and more "brute force" than would be natural for a pure-CPU intersection algorithm. For comparison, we have made C-code that mimics the GPU-algorithm. This may seem to favor the GPU over the CPU, but the effect is lessened by the fact that our GPU-algorithm will be even more efficient when combined with proper controlling CPU-based code. Such code will add more adaptivity to the GPUalgorithm. Work is in progress to implement a CPU+GPU-based module that

Table 1. Timings in seconds for a selection of sizes $n$. For small $n$, computational overhead is significant, but we see convergence in the speedup factor quickly after. This also testifies to the scalability of the algorithm and code.

\begin{tabular}{rrrr}
$n$ & GPU & CPU & Speedup factor \\
\hline 4 & $7.456 \mathrm{e}-03$ & $6.831 \mathrm{e}-03$ & 0.9 \\
5 & $1.138 \mathrm{e}-02$ & $7.330 \mathrm{e}-02$ & 6.4 \\
6 & $7.271 \mathrm{e}-02$ & $1.043 \mathrm{e} 00$ & 14.3 \\
7 & $9.573 \mathrm{e}-01$ & $1.607 \mathrm{e} 01$ & 16.8 \\
8 & $1.515 \mathrm{e} 01$ & $2.555 \mathrm{e} 02$ & 16.9
\end{tabular}
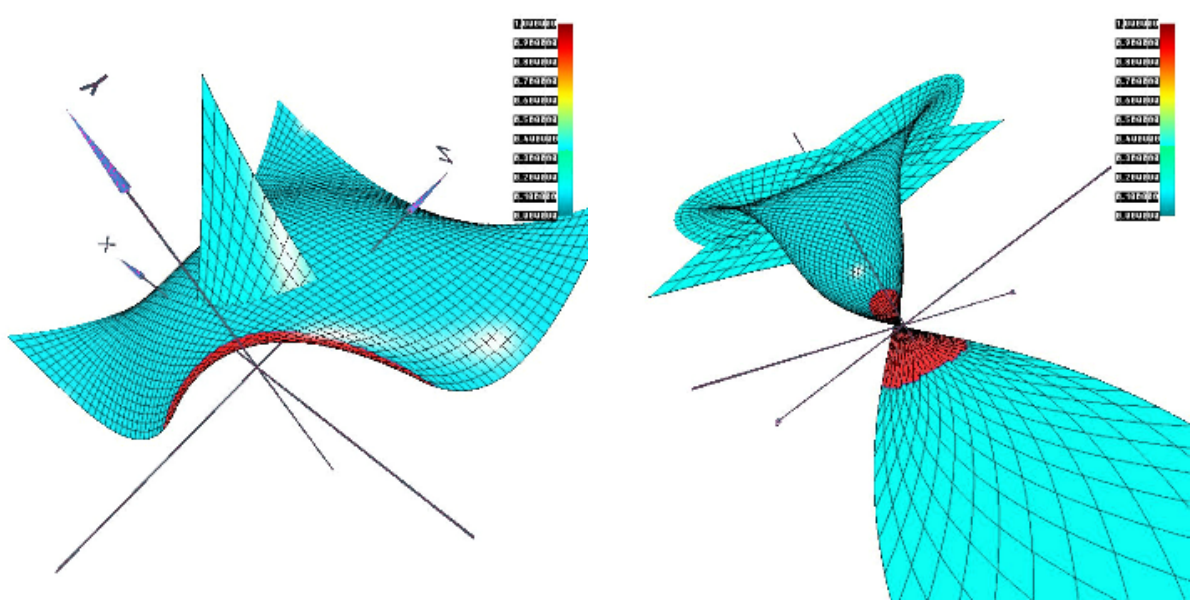

Fig. 4. A cubic Bezier patch with one corner pulled through itself, to create a (transversal) self-intersection. This also creates a crease in the surface, a region with high curvature. If the corner was pulled even more, this fold would produce a self-intersection with degenerate normals. As it is, the normals are only "near degenerate", and the region (darkened) has been detected by our GPU code, given a sufficiently high tolerance for "degeneracy". To the left, the Bezier patch, to the right, the corresponding normal surface, where the near degenerate normals appear as a region around the origin. 
can be substituted for a corresponding purely CPU-based module in a widely used CAD system, giving us an even better test bed.

On an NVIDIA GeForce 7800GT graphics card, we have tested the subdivision of cubic Bezier patches into $2^{n} \times 2^{n}$ subpatches, followed by tests for degenerate normals, subdivision of the quintic normal patch into the same number of normal subpatches, computation of the approximate normal cones, the bounding boxes, and finally the bounding box pair intersections. The CPU-version (C-code) was run on an AMD X2 4400+ without threading, and is compiled with the GNU gcc compiler with optimization -02 . Tests with $-03,-$ march=k8, - mf pmath=sse , 387 and -ffast-math did not produce significantly different timings. Explicit vector instructions are not used. The corresponding times are listed in Table 1 . An illustration of the detection of near degenerate normals in a near-self-intersection situation is shown in Figure 4.

\section{References}

1. Dokken T., Aspect of Intersection algorithms and Approximation, Thesis for the doctor philosophias degree, University of Oslo, Norway, 1997, 52-105.

2. Farin, G.: Curves and surfaces for CAGD: a practical guide. Morgan Kaufmann Publishers Inc. San Francisco, CA, USA (2002)

3. Hohmeyer, M. E., Robust and Efficient Surface Intersection for Solid Modelling, Report No. UCB/CSD 92/681, Computer Science Division, University of California, (1992).

4. Horn, D.: Stream Reduction Operations for GPGPU Applications, in GPUGems 2 : Programming Techniques for High-Performance Graphics and General-Purpose Computation, Addison-Wesley, (2005) 573-587

5. Patrikalakis, N.M.: Shape Interrogation for Computer Aided Design and Manufacturing. Springer-Verlag New York, Inc. Secaucus, NJ, USA (2002)

6. Sederberg, T.W. and A.K. Zundel, Pyramides that bound surface patches. CVGIP: Graphics Models and Image Processing, (1996), 75-81.

7. Sinha, P., E. Klassen and K.K. Wang, Exploiting topological and geometric properties for selective subdivision. In ACM Symposium on Computational Geometry, ACM Press, (1985), 39-45.

8. Skytt, V., A recursive approach to surface-surface intersection, in Mathematical Methods for Curves and Surfaces: Tromsø 2004, M. Dæhlen, K. Mørken, and L. L. Schumaker (eds.), Nashboro Press, Brentwood, (2005), 3272014338.

9. Skytt, V., Challenges in surface-surface intersections, in Computational Methods for Algebraic Spline Surfaces (COMPASS), T. Dokken and B. Jüttler (eds), Springer, (2004), 11-26. 